\title{
Improved Deterministic $N$-To-One Joint Remote Preparation of an Arbitrary Qubit via EPR Pairs
}

\author{
Wen-Jie Liu · Zheng-Fei Chen · Chao \\ Liu · Yu Zheng
}

Received: date / Accepted: date

\begin{abstract}
Recently, Bich et al. (Int. J. Theor. Phys. 51: 2272, 2012) proposed two deterministic joint remote state preparation (JRSP) protocols of an arbitrary single-qubit state: one is for two preparers to remotely prepare for a receiver by using two Einstein-Podolsky-Rosen (ERP) pairs; the other is its generalized form in the case of arbitrary $N(N>2)$ preparers via $N$ ERP pairs. While examining these two protocols, we find that the success probability for the receiver achieving the desired state is not deterministic, i.e., $P_{\text {suc }}^{N>2}<1$, for $N>2$ preparers in the second protocol. Through constructing two sets of adaptive projective measurement bases for both the real space and the complex space, an improved deterministic $N$-to-one JRSP protocol for an arbitrary single-qubit state is presented. Analysis shows our protocol can truly achieve the unit success probability, i.e., $P_{s u c}^{N \geq 2}=1$. What is more, the receiver can be randomly assigned even after the distribution of the qubits of EPR pairs, so it is more flexible and applicable in the network situation.
\end{abstract}

Keywords Joint remote state preparation $\cdot N$-to-one $\cdot$ EPR pairs $\cdot$ Projective measurement basis - Unit success probability

\section{Introduction}

With the rapid development of quantum mechanics in recent decades, many protocols of quantum information have been flourished by utilizing quantum

\footnotetext{
W.-J. Liu · Y. Zheng

Jiangsu Engineering Center of Network Monitoring, Nanjing University of Information Science \& Technology, Nanjing 210044, P.R.China
}

E-mail: wenjiel@163.com

W.-J. Liu · Z.-F Chen · C. Liu · Y. Zheng

School of Computer and Software, Nanjing University of Information Science \& Technology, Nanjing 210044, P.R.China 
mechanics principles, including quantum key distribution (QKD) [1-3], quantum secret sharing (QSS) [4-6], quantum direct communication (QDC) [7-9], quantum teleportation (QT) [10-12], quantum private comparison (QPC) [1315], and so on. In the recent ten years, a new direction, remote state preparation (RSP) [16-18], has become a hot topic in the quantum information filed. Similar to QT, in RSP the preparer can exploit the nonlocal correlation of the quantum entangled state shared in advance to prepare the original state in the remote place. But the main difference is that the preparer must know all the information of the state in RSP, while in QT the preparer knows nothing about the state.

The early RSP protocols always focus on one preparer and one receiver, and the preparer knows all the information of the prepared state. However, for some highly sensitive and important information, it might be unreliable to let one preparer hold everything. To overcome this defection, joint remote state preparation (JRSP) was put forward. The pioneering JRSP protocol was proposed by Xia et al. [19] in 2007, in which the authors realize the multiparty remote preparation of the quantum state $(\alpha|0\rangle \otimes M+\beta|1\rangle \otimes M, M=1,2, \ldots, \infty)$ with unit fidelity but less than unit probability between $N-1$ senders and one receiver using one $N$-particle non-maximally entangled Greenberger-HorneZeilinger (GHZ) state as the quantum channel. Since then, JRSP has attracted a lot of attention in recent years [20-25]. Unfortunately, these protocols are probabilistic, i.e., they cannot be realized with unit success probability.

To ensure the unit success probability of JRSP, a new direction of JRSP, namely deterministic JRSP, has been put forward. In 2011, Xiao et al. [26] introduced a three-step strategy of JRSP to remotely prepare an arbitrary twoand three-qubit state by using GHZ states as the shared quantum resource. In this protocol, two preparers measure their qubits orderly rather than independently, and the success probability of preparation can be increased to 1 . Inspired by the three-step strategy, many other deterministic JRSP protocols have been proposed [27-30]. For example, In 2012, An et al. [27] put forward a scheme to deterministically prepare the most general single- and two-qubit state by using four Einstein-Podolsky-Rosen (EPR) pairs as the shared quantum resource. And then, Chen et al. [28] proposed a scheme to joint remotely prepare an arbitrary three-qubit state deterministically by using six EPR pairs. In 2013, Wang et al. [29] proposed a scheme to jointly and remotely prepare an arbitrary two-qubit state, and generalize it to the arbitrary three-qubit case. Compared with Refs. [27] and [28], Wang et al.'s protocol just requires two EPR pairs and one GHZ state in the arbitrary two-qubit case and four EPR pairs and one GHZ states in the arbitrary three-qubit case. Being more robust and persistent than GHZ states, Cluster states are also utilized in JRSP. In 2013, Wang et al. [30] proposed a new version of deterministic JRSP protocol for an arbitrary two-qubit state by using the six-qubit cluster state. However, To the authors knowledge, most of deterministic JRSP protocols only focus on the two-preparer case, and these protocols are impossible or difficult to be directly generalized to the arbitrary preparers case. 
Recently, Bich et al. [31] proposed two deterministic JRSP protocols using ERP pairs: one is for two preparers to remotely prepare arbitrary single-qubit state; and the other is for $N(N>2)$ preparers. The authors claim that the total success probability is $1\left(P_{\text {suc }}=1\right)$ both for two preparers and $N>2$ preparers. Unfortunately, we find it is not true in case of $N>2$ preparers: the success probability $P_{s u c}^{N>2}<1$. In order to solve the problem, we tactfully constructed two sets of projective measurement bases, i.e., the real-coefficient measurement basis and the complex-coefficient measurement basis, and further proposed an $N$-to-one JRSP protocol for an arbitrary single-qubit state with unit success probability (i.e, $P_{s u c}^{N \geq 2}=1$ ).

The paper is organized as follows. In the next section, Bich et al.'s second protocols, especially the second protocol, are briefly reviewed and its correctness analysis is given in detail. In Sect. 3, two sets of projective measurement bases are constructed, and based on them, an improved deterministic $N$-toone JRSP protocol of an arbitrary single-qubit state is proposed. Finally, a concise summary is rendered in Sect. 4.

\section{Review of Bich et al.'s protocols and the correctness analysis}

\subsection{Review of Bich et al.'s protocols}

In Bich et al.'s first protocol, they proposed a deterministic JRSP protocol wtih $N=2$ preparers and a receiver. In this protocol, two preparers, Alice and Bob, can help the remote receiver, Charlie, prepare a single-qubit state

$$
|\phi\rangle=a|0\rangle+b^{i \varphi}|1\rangle,
$$

where coefficients $a, b$ are real with the normalization condition $|a|^{2}+|b|^{2}=1$, and $\varphi \in[0,2 \pi]$. To our knowledge, the success probability for the receiver achieving the desired state is truly 1 , so we just skip the detail of Bich et al.'s first protocol. In the appendix, Bich et al. propose the second protocol with $N>2$ preparers, in which the authors tried to directly generalize the first protocol to the situation of $N>2$ preparers, but unfortunately, there exists a fatal problem. Before reviewing the procedures of Bich et al.'s second protocol, we need have some knowledge about the pre-shared quantum channel among $N$ preparers (Alice, Bob 1, Bob 2, ..., Bob $N$ ) and a receiver (Charlie) as shown in Fig. 1.

For the convenience of description, the authors took $N=3$ (Alice, Bob 1, and Bob 2), and the participants pre-share three EPR pairs as the quantum channel

$$
|Q\rangle_{A_{1} B_{1} A_{2} B_{2} A_{3} C}=|E P R\rangle_{A_{1} B_{1}}|E P R\rangle_{A_{2} B_{2}}|E P R\rangle_{A_{3} C},
$$

where $|E P R\rangle=\frac{1}{\sqrt{2}}(|00\rangle+|11\rangle)$, qubits $A_{1}, A_{2}, A_{3}$ are hold by Alice, and qubits $B_{1}, B_{2}, C$ by Bob 1 , Bob 2 and Charlie, respectively. Alice is allowed to know $\{a, b\}$, Bob 1 and Bob 2 share the knowledge of $\varphi$ in the following 


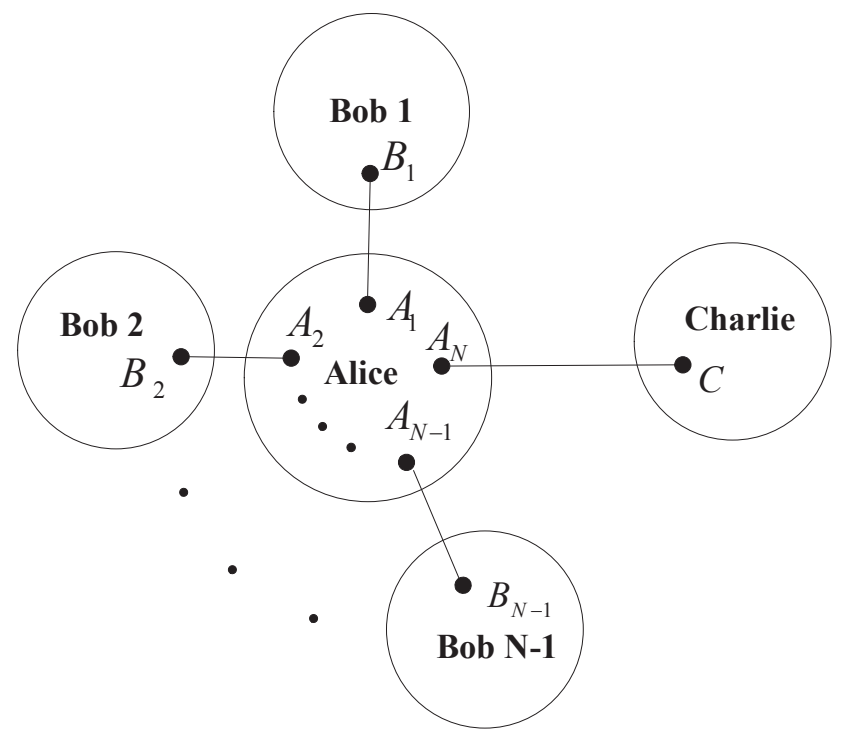

Fig. 1: ${ }^{31]}$ The qubits distribution for JRSP of the most general single-qubit state via $N$ EPR pairs for the situation of $N$ preparers (Alice, Bob 1, Bob 2, ... and Bob N-1) and a receiver (Charlie). Qubits are represented by dots and entangled qubits are connected by a solid line.

way: Bob 1 knows $\varphi_{1}$ and Bob 2 knows $\varphi_{2}$, and $\varphi=\varphi_{1}+\varphi_{2}$. The detailed three-step protocol for $N=3$ preparers can be described as below.

Step 1. Alice measures her three qubits in the basis $\left\{\left|u_{k l m}\right\rangle_{A_{1} A_{2} A_{3}} ; k, l, m \in\right.$ $\{0,1\}\}$,

$$
\left(\begin{array}{l}
\left|u_{000}\right\rangle_{A_{1} A_{2} A_{3}} \\
\left|u_{001}\right\rangle_{A_{1} A_{2} A_{3}} \\
\left|u_{010}\right\rangle_{A_{1} A_{2} A_{3}} \\
\left|u_{011}\right\rangle_{A_{1} A_{2} A_{3}} \\
\left|u_{100}\right\rangle_{A_{1} A_{2} A_{3}} \\
\left|u_{101}\right\rangle_{A_{1} A_{2} A_{3}} \\
\left|u_{110}\right\rangle_{A_{1} A_{2} A_{3}} \\
\left|u_{111}\right\rangle_{A_{1} A_{2} A_{3}}
\end{array}\right)=\left(\begin{array}{cccccccc}
a & 0 & 0 & 0 & 0 & 0 & 0 & b \\
b & 0 & 0 & 0 & 0 & 0 & 0 & -a \\
0 & a & 0 & 0 & 0 & 0 & b & 0 \\
0 & b & 0 & 0 & 0 & 0 & -a & 0 \\
0 & 0 & a & 0 & 0 & b & 0 & 0 \\
0 & 0 & b & 0 & 0 & -a & 0 & 0 \\
0 & 0 & 0 & a & b & 0 & 0 & 0 \\
0 & 0 & 0 & -b & a & 0 & 0 & 0
\end{array}\right)\left(\begin{array}{l}
|000\rangle_{A_{1} A_{2} A_{3}} \\
|001\rangle_{A_{1} A_{2} A_{3}} \\
|010\rangle_{A_{1} A_{2} A_{3}} \\
|011\rangle_{A_{1} A_{2} A_{3}} \\
|100\rangle_{A_{1} A_{2} A_{3}} \\
|101\rangle_{A_{1} A_{2} A_{3}} \\
|110\rangle_{A_{1} A_{2} A_{3}} \\
|111\rangle_{A_{1} A_{2} A_{3}}
\end{array}\right),
$$

and publicly broadcasts the measurement outcome to Bob 1, Bob 2 and Charlie. Expressing the quantum channel $|Q\rangle_{A_{1} B_{1} A_{2} B_{2} A_{3} C}$ through $\left|u_{k l m}\right\rangle_{A_{1} A_{2} A_{3}}$,

$$
|Q\rangle_{A_{1} B_{1} A_{2} B_{2} A_{3} C}=\frac{1}{2 \sqrt{2}} \sum_{m=0}^{1} \sum_{l=0}^{1} \sum_{k=0}^{1}\left|u_{k l m}\right\rangle_{A_{1} A_{2} A_{3}}\left|L_{k l m}\right\rangle_{B_{1} B_{2} C},
$$

we derive $\left|L_{k l m}\right\rangle_{B_{1} B_{2} C}$ in the form

$$
\left|L_{000}\right\rangle_{B_{1} B_{2} C}=a|000\rangle_{B_{1} B_{2} C}+b|111\rangle_{B_{1} B_{2} C},
$$




$$
\begin{aligned}
& \left|L_{001}\right\rangle_{B_{1} B_{2} C}=-a|111\rangle_{B_{1} B_{2} C}+b|000\rangle_{B_{1} B_{2} C}, \\
& \left|L_{010}\right\rangle_{B_{1} B_{2} C}=a|001\rangle_{B_{1} B_{2} C}+b|110\rangle_{B_{1} B_{2} C}, \\
& \left|L_{011}\right\rangle_{B_{1} B_{2} C}=-a|110\rangle_{B_{1} B_{2} C}+b|001\rangle_{B_{1} B_{2} C}, \\
& \left|L_{100}\right\rangle_{B_{1} B_{2} C}=a|010\rangle_{B_{1} B_{2} C}+b|101\rangle_{B_{1} B_{2} C} \\
& \left|L_{101}\right\rangle_{B_{1} B_{2} C}=-a|101\rangle_{B_{1} B_{2} C}+b|010\rangle_{B_{1} B_{2} C}, \\
& \left|L_{110}\right\rangle_{B_{1} B_{2} C}=a|011\rangle_{B_{1} B_{2} C}+b|100\rangle_{B_{1} B_{2} C} \\
& \left|L_{111}\right\rangle_{B_{1} B_{2} C}=a|100\rangle_{B_{1} B_{2} C}-b|011\rangle_{B_{1} B_{2} C}
\end{aligned}
$$

Step 2. Bob 1 and Bob 2 independently measure their qubits. If $k l m=000$ or 010, each Bob $j(j=1,2)$ uses a measurement basis determined by

$$
\left(\begin{array}{c}
\left|v_{0}\right\rangle_{B_{j}} \\
\left|v_{1}\right\rangle_{B_{j}}
\end{array}\right)=V^{(0)}\left(\varphi_{j}\right)\left(\begin{array}{c}
|0\rangle_{B_{j}} \\
|1\rangle_{B_{j}}
\end{array}\right) ;
$$

If $k l m=001$ or 011 , the basis for each Bob $j$ is

$$
\left(\begin{array}{l}
\left|v_{0}\right\rangle_{B_{j}} \\
\left|v_{1}\right\rangle_{B_{j}}
\end{array}\right)=V^{(1)}\left(\varphi_{j}\right)\left(\begin{array}{c}
|0\rangle_{B_{j}} \\
|1\rangle_{B_{j}}
\end{array}\right)
$$

where

$$
V^{(r)}(\varphi)=\frac{1}{\sqrt{2}}\left(\begin{array}{cc}
1 & e^{-(-1)^{r} i \varphi} \\
e^{(-1)^{r} i \varphi} & -1
\end{array}\right), r \in\{0,1\} .
$$

In case $k l m=100$ or 110 , Bob 1 uses a measurement basis determined by Eq.(13), and the basis for Bob 2 is Eq. (14); while $k l m=101$ or 111, the bases for Bob 1 and Bob 2 are determined by Eq. (14) and (13), respectively. After measurement, Bob 1 and Bob 2 announce their measurement outcomes $n, s$ $(n, s \in\{0,1\})$ to Charlie.

Step 3. Charlie converts the qubit $C$ to be the desired state $|\phi\rangle_{C}$ by applying reconstruction operators $R_{k l m n s}$. The operators $R_{k l m n s}$ that Charlie needs in this step are shown in Table 1.

Table 1: ${ }^{[31]}$ The reconstruction operator $R_{k l m n s}$, conditioned on the measurement outcomes $k l m, n$ and $s$ of Alice, Bob 1 and Bob 2, respectively.

$I$ is the identity operator, $X=|0\rangle\langle 1|+| 1\rangle\langle 0|$ the bit-flip operator and $Z=|0\rangle\langle 0|-| 1\rangle\langle 1|$ the phase-flip one

\begin{tabular}{lll}
\hline$\sharp$ & klmns & $R_{\text {klmns }}$ \\
\hline $1-8$ & $00000,00011,10000,10011,01101,01110,11101$ or 11100 & $I$ \\
$9-16$ & $01000,01011,11000,11011,00101,00110,10101$ or 10110 & $X$ \\
$17-24$ & $00100,00111,10100,10111,01001,01010,11001123$ or 11010 & $Z X$ \\
$25-32$ & $01100,01111,11100,11111,00001,00010,10001$ or 10010 & $Z$ \\
\hline
\end{tabular}




\subsection{Correctness analysis}

In Ref. [31], the authors claimed the receiver can get the desirable state with the unit success probability $\left(P_{\text {suc }}=1\right)$ both for two preparers and $N>2$ preparers. In fact, it is not true in the case of $N>2$ preparers. In the following, we will analyze its correctness in detail.

In Step 1, Alice measures her three qubits in the basis $\left\{\left|u_{k l m}\right\rangle_{A_{1} A_{2} A_{3}} ; k, l, m \in\right.$ $\{0,1\}\}$ and publishes her outcome to the other participants (Bob 1, Bob 2 and Charlie). For sake of clearness, we assume the measurement outcome is $\mathrm{klm}=$ 010, and according to the selection strategy of measurement basis in Step 2, Bob 1 and Bob 2 will use the measurement basis of Equation (13), i.e., the basis for Bob 1 is $\left(\begin{array}{l}\left|v_{0}\right\rangle_{B_{1}} \\ \left|v_{1}\right\rangle_{B_{1}}\end{array}\right)=V^{0}\left(\varphi_{1}\right)\left(\begin{array}{l}|0\rangle_{B_{1}} \\ |1\rangle_{B_{1}}\end{array}\right)=\frac{1}{\sqrt{2}}\left(\begin{array}{cc}1 & e^{-i \varphi_{1}} \\ e^{i \varphi_{1}} & -1\end{array}\right)\left(\begin{array}{l}|0\rangle_{B_{1}} \\ |1\rangle_{B_{1}}\end{array}\right)$, and the basis for Bob 2 is $\left(\begin{array}{l}\left|v_{0}\right\rangle_{B_{2}} \\ \left|v_{1}\right\rangle_{B_{2}}\end{array}\right)=V^{0}\left(\varphi_{2}\right)\left(\begin{array}{c}|0\rangle_{B_{2}} \\ |1\rangle_{B_{2}}\end{array}\right)=\frac{1}{\sqrt{2}}\left(\begin{array}{cc}1 & e^{-i \varphi_{2}} \\ e^{i \varphi_{2}} & -1\end{array}\right)\left(\begin{array}{l}|0\rangle_{B_{2}} \\ |1\rangle_{B_{2}}\end{array}\right)$. Then, $\left|L_{010}\right\rangle_{B_{1} B_{2} C}$ can be rewritten as

$$
\begin{aligned}
\left|L_{010}\right\rangle_{B_{1} B_{2} C} & =\frac{1}{2} \sum_{n=0}^{1} \sum_{s=0}^{1}\left|v_{n}\right\rangle_{B_{1}}\left|v_{s}\right\rangle_{B_{2}}|\phi\rangle_{C} \\
& =\frac{1}{2}\left|v_{0}\right\rangle_{B_{1}}\left|v_{0}\right\rangle_{B_{2}}\left(a|1\rangle+b e^{i\left(\varphi_{1}+\varphi_{2}\right)}|0\rangle\right)_{C} \\
& +\frac{1}{2}\left|v_{0}\right\rangle_{B_{1}}\left|v_{1}\right\rangle_{B_{2}}\left(a e^{-i \varphi_{2}}|1\rangle-b e^{i \varphi_{1}}|0\rangle\right)_{C} \\
& +\frac{1}{2}\left|v_{1}\right\rangle_{B_{1}}\left|v_{0}\right\rangle_{B_{2}}\left(a e^{-i \varphi_{1}}|1\rangle-b e^{i \varphi_{2}}|0\rangle\right)_{C} \\
& +\frac{1}{2}\left|v_{1}\right\rangle_{B_{1}}\left|v_{1}\right\rangle_{B_{2}}\left(a e^{-i\left(\varphi_{1}+\varphi_{2}\right)}|1\rangle+b|0\rangle\right)_{C}
\end{aligned}
$$

Obviously, only when $n s=00$, qubit $C$ will collapse into $|\phi\rangle_{C}=a|1\rangle+$ $b e^{i\left(\varphi_{1}+\varphi_{2}\right)}|0\rangle$, and the receiver can achieve the desirable state just with the identity operator. But if $n s=01\left(|\phi\rangle_{C}=a e^{-i \varphi_{2}}|1\rangle-b e^{i \varphi_{1}}|0\rangle\right), 10\left(|\phi\rangle_{C}=\right.$ $\left.a e^{-i \varphi_{1}}|1\rangle-b e^{i \varphi_{2}}|0\rangle\right)$ or $11\left(|\phi\rangle_{C}=a e^{-i\left(\varphi_{1}+\varphi_{2}\right)}|1\rangle+b|0\rangle\right)$, there is no any appropriate unitary operator that can be adopted to transform the state $|\phi\rangle_{C}$ to the desirable state. That is to say, the probability for Charlie finding the desirable state is $1 / 4$ under this situation.

In the general case, there are eight possible outcomes $\{000,001,010,011,100$, $101,110,111\}$ for Alice, and the probability of each outcome is equal. That means, the probability for Alice obtaining each state $\left|u_{k l m}\right\rangle_{A_{1} A_{2} A_{3}}(k, l, m=$ $0,1)$ is $1 / 8$. Similar to the case of $k l m=010$, Charlie can also obtain the desirable state with the probability of $1 / 4$ in the other seven cases. So, the total success probability is

$$
P_{\text {suc }}^{N=3}=\left(\frac{1}{8} \times \frac{1}{4}\right) \times 8=\frac{1}{4}<1 .
$$

Clearly, this is contrary to what the authors claimed: "the total success probability is obviously 1" [31]. 


\section{Improved deterministic $N$-to-one JRSP of an arbitrary qubit}

3.1 The construction of measurement basis

The construction of measurement basis is at the heart of RSP or JRSP, and it is the key point that will influence the final success probability of obtaining the desirable state. In order to improve the success probability of the $N$-toone JRSP to 1 , the first task is to tactfully construct two suitable sets of measurement bases, the real-coefficient measurement basis and the complexcoefficient one.

In our JRSP protocol, we will use the projective measurements to measure the qubits. Before constructing these ingenious projective measurement bases, we need have some knowledge of projective measurement.

Theorem 1 Projective measurement ${ }^{[32]}$ : A projective measurement is described by an observable, $M$, a Hermitian operator on the state space of the system being observed. The observable has a spectral decomposition,

$$
M=\sum_{m} m P_{m}
$$

where $P_{m}$ is the projector onto the eigenspace of $M$ with eigenvalue $m$. The possible outcomes of the measurement correspond to the eigenvalues, $m$, of the observable.

According to the definition of projective measurement (Theorem 1), projective measurement can be understood as a special case of Postulate 3 (i.e., the quantum measurement postulate, seen in Refs. [32] and [33]). Specifically, a complete set of orthogonal projectors $P_{m}$ satisfy the following relations: (1) $\sum_{m} P_{m}=I$; and (2) $P_{m} P_{m^{\prime}}=\delta_{m, m^{\prime}} P_{m}\left(\delta_{m, m^{\prime}}=1\right.$, if $m=m^{\prime} ; \delta_{m, m^{\prime}}=0$, if $\left.m \neq m^{\prime}\right)$. In quantum information field, a widely used phrase, to 'measure in a basis $|m\rangle$ ', where $|m\rangle$ form an orthonormal basis, simply means to perform the projective measurement with projectors as below

$$
P_{m}=|m\rangle\langle m| \text {. }
$$

In our protocol, Alice holds $N$ qubits $A_{1}, A_{2}, \ldots A_{N}$, and she knows the real part $\{a, b\}$ of the state (given in Eq. (1)). So the projection operators $\left\{P_{m}\right\}$ can be described as $P_{m}=\left|u_{l_{1}, l_{2}, \ldots, l_{N}}\right\rangle_{A_{1}, A_{2}, \ldots, A_{N}}\left\langle\left. u_{l_{1}, l_{2}, \ldots, l_{N}}\right|_{A_{1}, A_{2}, \ldots, A_{N}}\right.$ $\left(l_{1}, l_{2}, \ldots l_{N} \in\{0,1\}\right)$, where the set of states $\left\{\left|u_{l_{1}, l_{2}, \ldots l_{N}}\right\rangle_{\left.A_{1}, A_{2}, \ldots A_{N}\right\}}\right\}$ is a complete set of orthonormal basis and can be defined as

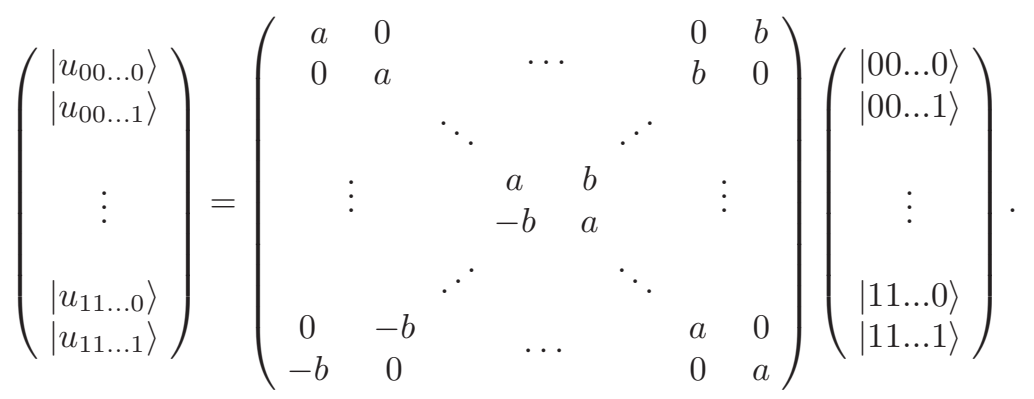


The Eq. (20) can be generalized to a collection $\left\{\left|u_{l_{1} l_{2} \ldots l_{N}}\right\rangle=a\left|l_{1} l_{2} \ldots l_{N}\right\rangle_{A_{1}, A_{2}, \ldots A_{N}}\right.$ $\left.+(-1)^{l_{1}} b\left|\overline{l_{1} l_{2}} \ldots \overline{l_{N}}\right\rangle_{A_{1}, A_{2}, \ldots A_{N}} \mid l_{k} \in\{0,1\}, \overline{l_{k}}=1-l_{k}, 1 \leq k \leq N\right\}$. Simultaneously, since each Bob $j(1 \leq j \leq N-1)$ holds one qubit $B_{j}$ and knows a part of complex part $\varphi_{j}$, so, the corresponding complete set of orthonormal basis can be expressed as

$$
\left(\begin{array}{c}
\left|v_{0}\right\rangle_{B_{j}} \\
\left|v_{1}\right\rangle_{B_{j}}
\end{array}\right)=V^{\prime}\left(l_{j}\right)\left(\varphi_{j}\right)\left(\begin{array}{c}
|0\rangle_{B_{j}} \\
|1\rangle_{B_{j}}
\end{array}\right)
$$

where $l_{j}$ represents the value of subscript in $\left|u_{l_{1} l_{2} \ldots l_{N}}\right\rangle, l_{j} \in\{0,1\}$, and

$$
\begin{aligned}
V^{\prime(0)}\left(\varphi_{j}\right) & =\frac{1}{\sqrt{2}}\left(\begin{array}{cc}
1 & e^{-i \varphi_{j}} \\
1 & -e^{-i \varphi_{j}}
\end{array}\right), \\
V^{\prime(1)}\left(\varphi_{j}\right) & =\frac{1}{\sqrt{2}}\left(\begin{array}{cc}
e^{-i \varphi_{j}} & 1 \\
-e^{-i \varphi_{j}} & 1
\end{array}\right) .
\end{aligned}
$$

\subsection{Our $N$-To-One JRSP protocol}

By utilizing the measurement bases constructed in Sect. 3.1, we present an improved deterministic $N$-to-one JRSP of an arbitrary qubit as below.

Prerequisite. Suppose preparers (Alice, Bob 1, Bob 2, ... and Bob N1) want to help the receiver Charlie prepare an arbitrary single-qubit state (given in Eq. (1)), and they share $N$ EPR pairs as quantum channel, which is described as

$$
\left.|Q\rangle_{A_{1} B_{1} A_{2} B_{2} \ldots A_{N} C}=|E P R\rangle_{A_{1} B_{1}}|E P R\rangle_{A_{2} B_{2} \ldots} \ldots P R\right\rangle_{A_{N-1} B_{N-1}}|E P R\rangle_{A_{N} C}
$$

Here, Alice is allowed to know $\{a, b\}$, Bob 1 , Bob $2, \ldots$ and Bob N-1 share the knowledge of $\varphi$, and each Bob $j(1 \leq j \leq N-1)$ knows $\varphi_{j}$, where $\varphi=\sum_{j=1}^{N-1} \varphi_{j}$.

Step 1. Alice measures her $N$ qubits with the basis (given in Eq. (20)) and publicly broadcasts the measuring outcome $l_{1} l_{2} \ldots l_{N}\left(l_{1}, l_{2}, \ldots l_{N} \in\{0,1\}\right)$ to Bob 1, Bob 2, . . Bob N-1 and Charlie.

Step 2. Each Bob $j$ independently measures her qubit $B_{j}$ in the basis conditioned on $\varphi_{j}$ and Alice's outcome $l_{j}$. Specifically, each Bob $j$ uses a basic measurement shown in Eq. (21). After measurement, each Bob $j$ announces her measurement outcome $m_{j}\left(m_{j} \in\{0,1\}\right)$ to Charlie.

Step 3. Charlie converts the qubit $C$ to be the desired state $|\phi\rangle_{C}$ by apply-

$$
\begin{aligned}
& \text { ing reconstruction operators } R^{R_{l_{1}}^{l_{2} \ldots l_{N}}} \underbrace{m_{1} m_{2} \ldots m_{N-1}}_{N-1}=Z \underbrace{l_{N} \oplus \underbrace{m_{1} \oplus m_{2} \oplus \ldots \oplus m_{N-1}}_{N-1}}_{N} \\
& \underbrace{l_{1} \oplus l_{2} \oplus \ldots \oplus l_{N}}_{X} .
\end{aligned}
$$




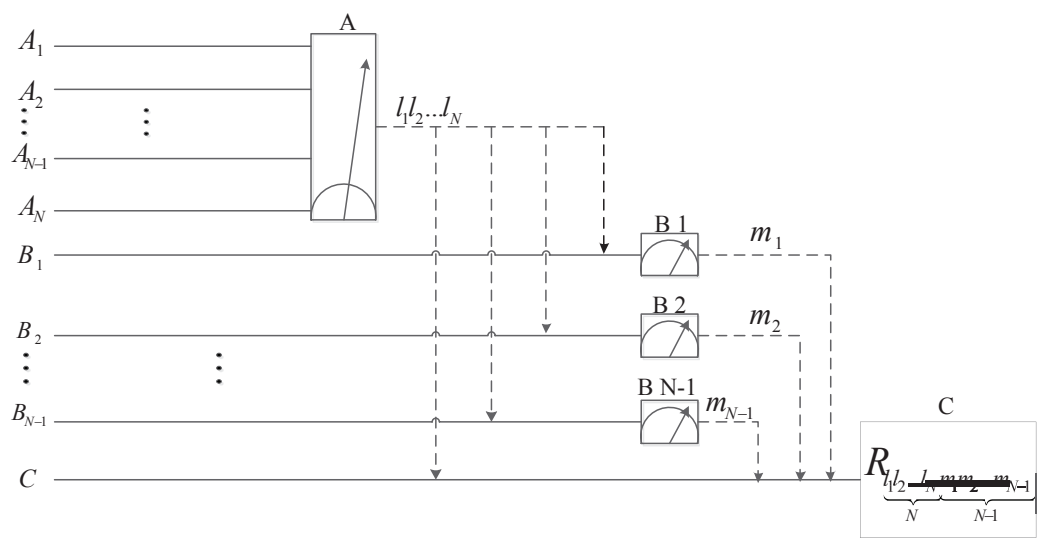

Fig. 2: The quantum circuit for deterministic deterministic $N$-to-one JRSP of an arbitrary qubit via $N$ EPR pairs. Particles $A_{1} A_{2} \ldots A_{N}$ are hold by Alice, $B_{1}, B_{2}, \ldots, B_{N-1}$ hold by Bob 1 , Bob $2, \ldots$ and Bob N-1, respectively, and $C$ hold by Charlie. The classical communications are represented as the dashed arrows, while the quantum channels are represented as the solid lines. what is more, boxes A, B1, B2, and B N-1 depict Alice's, Bob 1's, Bob 2's, ..., and Bob N-1's measurement operation, respectively, and the last box $C$ denotes the unitary operation Charlie carries out.

The whole process of our $N$-to-one JRSP protocol can also be described in a quantum circuit form as shown in Fig. 2. For clarity, we consider $N=3$ (Alice, Bob 1, Bob 2) as example, and $|Q\rangle_{A_{1} B_{1} A_{2} B_{2} A_{3} C}=|E P R\rangle_{A_{1} B_{1}}|E P R\rangle_{A_{2} B_{2}}|E P R\rangle_{A_{3} C}$ is the quantum channel. At first, Alice measures her three qubits in the basis $\left\{\left|u_{l_{1} l_{2} l_{3}}\right\rangle_{A_{1} A_{2} A_{3}} ; l_{1}, l_{2}, l_{3} \in\{0,1\}\right\}:$

$$
\left(\begin{array}{l}
\left|u_{000}\right\rangle_{A_{1} A_{2} A_{3}} \\
\left|u_{001}\right\rangle_{A_{1} A_{2} A_{3}} \\
\left|u_{010}\right\rangle_{A_{1} A_{2} A_{3}} \\
\left|u_{011}\right\rangle_{A_{1} A_{2} A_{3}} \\
\left|u_{100}\right\rangle_{A_{1} A_{2} A_{3}} \\
\left|u_{101}\right\rangle_{A_{1} A_{2} A_{3}} \\
\left|u_{110}\right\rangle_{A_{1} A_{2} A_{3}} \\
\left|u_{111}\right\rangle_{A_{1} A_{2} A_{3}}
\end{array}\right)=\left(\begin{array}{cccccccc}
a & 0 & 0 & 0 & 0 & 0 & 0 & b \\
0 & a & 0 & 0 & 0 & 0 & b & 0 \\
0 & 0 & a & 0 & 0 & b & 0 & 0 \\
0 & 0 & 0 & a & b & 0 & 0 & 0 \\
0 & 0 & 0 & -b & a & b & 0 & 0 \\
0 & 0 & -b & 0 & 0 & a & 0 & 0 \\
0 & -b & 0 & 0 & 0 & 0 & a & 0 \\
-b & 0 & 0 & 0 & 0 & 0 & 0 & a
\end{array}\right)\left(\begin{array}{l}
|000\rangle_{A_{1} A_{2} A_{3}} \\
|001\rangle_{A_{1} A_{2} A_{3}} \\
|010\rangle_{A_{1} A_{2} A_{3}} \\
|011\rangle_{A_{1} A_{2} A_{3}} \\
|100\rangle_{A_{1} A_{2} A_{3}} \\
|101\rangle_{A_{1} A_{2} A_{3}} \\
|110\rangle_{A_{1} A_{2} A_{3}} \\
|111\rangle_{A_{1} A_{2} A_{3}}
\end{array}\right),
$$

and publicly broadcasts outcome $l_{1} l_{2} l_{3}$. Before measurement, the quantum channel can be rewritten as

$$
|Q\rangle_{A_{1} B_{1} A_{2} B_{2} A_{3} C}=\frac{1}{2 \sqrt{2}} \sum_{l_{1}=0}^{1} \sum_{l_{2}=0}^{1} \sum_{l_{3}=0}^{1}\left|u_{l_{1} l_{2} l_{3}}\right\rangle_{A_{1} A_{2} A_{3}}\left|L_{l_{1} l_{2} l_{3}}\right\rangle_{B_{1} B_{2} C} .
$$

And we derive $\left|L_{l_{1} l_{2} l_{3}}\right\rangle_{B_{1} B_{2} C}$ in the form

$$
\left|L_{l_{1} l_{2} l_{3}}\right\rangle_{B_{1} B_{2} C}=a\left|l_{1} l_{2} l_{3}\right\rangle_{B_{1} B_{2} C}+(-1)^{l_{1}} b\left|\overline{l_{1} l_{2} l_{3}}\right\rangle_{B_{1} B_{2} C} .
$$


After Alice's measurement, Bob 1 and Bob 2 use the basis to measure their own qubit. The state in Eq. (27) can be expressed as

$$
\left|L_{l_{1} l_{2} l_{3}}\right\rangle_{B_{1} B_{2} C}=\frac{1}{2} \sum_{m_{1}=1}^{2} \sum_{m_{2}=1}^{2}\left|v_{m_{1}}\right\rangle_{B_{1}}\left|v_{m_{2}}\right\rangle_{B_{2}}|\phi\rangle_{C} .
$$

We assume $l_{1} l_{2} l_{3}=010$ without generality, then Bob 1 and Bob 2 choose the basis $\left\{\left|v_{m_{1}}\right\rangle_{B_{1}} \mid m_{1} \in\{0,1\}\right\}$

$$
\left(\begin{array}{l}
\left|v_{0}\right\rangle_{B_{1}} \\
\left|v_{1}\right\rangle_{B_{1}}
\end{array}\right)=\frac{1}{\sqrt{2}}\left(\begin{array}{cc}
1 & e^{-i \varphi_{1}} \\
1 & -e^{-i \varphi_{1}}
\end{array}\right)\left(\begin{array}{l}
|0\rangle_{B_{1}} \\
|1\rangle_{B_{1}}
\end{array}\right),
$$

and $\left\{\left|v_{m_{2}}\right\rangle_{B_{2}} \mid m_{2} \in\{0,1\}\right\}$ :

$$
\left(\begin{array}{l}
\left|v_{0}\right\rangle_{B_{2}} \\
\left|v_{1}\right\rangle_{B_{2}}
\end{array}\right)=\frac{1}{\sqrt{2}}\left(\begin{array}{cc}
e^{-i \varphi_{2}} & 1 \\
-e^{-i \varphi_{2}} & 1
\end{array}\right)\left(\begin{array}{l}
|0\rangle_{B_{2}} \\
|1\rangle_{B_{2}}
\end{array}\right),
$$

respectively. We can rewritten $\left|L_{010}\right\rangle_{B_{1} B_{2} C}$ as

$$
\begin{aligned}
\left|L_{010}\right\rangle_{B_{1} B_{2} C} & =\frac{1}{2} a\left(\left|v_{0}\right\rangle_{B_{1}}+\left|v_{1}\right\rangle_{B_{1}}\right)\left(\left|v_{0}\right\rangle_{B_{2}}+\left|v_{1}\right\rangle_{B_{2}}\right)|0\rangle_{C} \\
& +\frac{1}{2} b e^{i\left(\varphi_{1}+\varphi_{2}\right)}\left(\left|v_{0}\right\rangle_{B_{1}}-\left|v_{1}\right\rangle_{B_{1}}\right)\left(\left|v_{0}\right\rangle_{B_{2}}-\left|v_{1}\right\rangle_{B_{2}}\right)|1\rangle_{C} \\
& =\frac{1}{2}\left|v_{0}\right\rangle_{B_{1}}\left|v_{0}\right\rangle_{B_{2}}\left(a|0\rangle+b e^{i\left(\varphi_{1}+\varphi_{2}\right)}|1\rangle\right)_{C} \\
& +\frac{1}{2}\left|v_{0}\right\rangle_{B_{1}}\left|v_{1}\right\rangle_{B_{2}}\left(a|0\rangle-b e^{i\left(\varphi_{1}+\varphi_{2}\right)}|1\rangle\right)_{C} \\
& +\frac{1}{2}\left|v_{1}\right\rangle_{B_{1}}\left|v_{0}\right\rangle_{B_{2}}\left(a|0\rangle-b e^{i\left(\varphi_{1}+\varphi_{2}\right)}|1\rangle\right)_{C} \\
& +\frac{1}{2}\left|v_{1}\right\rangle_{B_{1}}\left|v_{1}\right\rangle_{B_{2}}\left(a|0\rangle+b e^{i\left(\varphi_{1}+\varphi_{2}\right)}|1\rangle\right)_{C} .
\end{aligned}
$$

Easy to see, whatever the outcome is, Charlie can achieve the desirable state by applying some reconstruction unitary operator $R_{l_{1} l_{2} l_{3} m_{1} m_{2}}$ (all of which are shown in Table 2), and the success probability in each cases is 1 . Moreover, the probability of obtaining the state $\left|L_{l_{1} l_{2} l_{3}}\right\rangle_{B_{1} B_{2} C}$ for each of the outcomes $\left\{l_{1} l_{2} l_{3}\right\}$ is equal. So, the total success probability $P_{s u c}^{N \geq 2}$ can be calculated as below

$$
P_{\text {suc }}^{N \geq 2}=\left(\frac{1}{8} \times 1\right) \times 8=1 .
$$

\section{Summary}

In this paper, through analyzing Bich et al.'s second protocol with $N>2$ preparers, we find that the success probability $P_{\text {suc }}^{N>2}=1 / 4<1$. In order to fix the drawback, we firstly constructed two sets of projective measurement bases: the real-coefficient basis $\left\{\left|u_{l_{1} l_{2} \ldots l_{N}}\right\rangle \mid l_{k} \in\{0,1\}\right\}$ and the complex-coefficient basis $\left\{\left|v_{0}\right\rangle_{B_{j}},\left|v_{1}\right\rangle_{B_{j}}\right\}$, and further proposed an improved deterministic $N$-to-one 
Table 2: The collapsed state $|\phi\rangle_{C}$ of Charlie's qubits $C$ and the corresponding recovery operators $R_{l_{1} l_{2} l_{3} m_{1} m_{2}}$ related to the measurement outcomes $\left(l_{1} l_{2} l_{3}, m_{1}, m_{2}\right)$ from Alice, Bob 1 and Bob 2

\begin{tabular}{|c|c|c|c|}
\hline$l_{1} l_{2} l_{3}$ & $m_{1} m_{2}$ & $|\phi\rangle_{C}$ & $R_{l_{1} l_{2} l_{3} m_{1} m_{2}}$ \\
\hline \multirow{4}{*}{000} & 00 & $a|0\rangle+b e^{i\left(\varphi_{1}+\varphi_{2}\right)}|1\rangle$ & $I$ \\
\hline & 01 & $a|0\rangle-b e^{i\left(\varphi_{1}+\varphi_{2}\right)}|1\rangle$ & $Z$ \\
\hline & 10 & $a|0\rangle-b e^{i\left(\varphi_{1}+\varphi_{2}\right)}|1\rangle$ & $Z$ \\
\hline & 11 & $a|0\rangle+b e^{i\left(\varphi_{1}+\varphi_{2}\right)}|1\rangle$ & $I$ \\
\hline \multirow{4}{*}{001} & 00 & $a|1\rangle+b e^{i\left(\varphi_{1}+\varphi_{2}\right)}|0\rangle$ & $X$ \\
\hline & 01 & $a|1\rangle-b e^{i\left(\varphi_{1}+\varphi_{2}\right)}|0\rangle$ & $Z X$ \\
\hline & 10 & $a|1\rangle-b e^{i\left(\varphi_{1}+\varphi_{2}\right)}|0\rangle$ & $Z X$ \\
\hline & 11 & $a|1\rangle+b e^{i\left(\varphi_{1}+\varphi_{2}\right)}|0\rangle$ & $X$ \\
\hline \multirow{4}{*}{010} & 00 & $a|0\rangle+b e^{i\left(\varphi_{1}+\varphi_{2}\right)}|1\rangle$ & $I$ \\
\hline & 01 & $a|0\rangle-b e^{i\left(\varphi_{1}+\varphi_{2}\right)}|1\rangle$ & $Z$ \\
\hline & 10 & $a|0\rangle-b e^{i\left(\varphi_{1}+\varphi_{2}\right)}|1\rangle$ & $Z$ \\
\hline & 11 & $a|0\rangle+b e^{i\left(\varphi_{1}+\varphi_{2}\right)}|1\rangle$ & $I$ \\
\hline \multirow{4}{*}{011} & 00 & $a|1\rangle+b e^{i\left(\varphi_{1}+\varphi_{2}\right)}|0\rangle$ & $X$ \\
\hline & 01 & $a|1\rangle-b e^{i\left(\varphi_{1}+\varphi_{2}\right)}|0\rangle$ & $Z X$ \\
\hline & 10 & $a|1\rangle-b e^{i\left(\varphi_{1}+\varphi_{2}\right)}|0\rangle$ & $Z X$ \\
\hline & 11 & $a|1\rangle+b e^{i\left(\varphi_{1}+\varphi_{2}\right)}|0\rangle$ & $X$ \\
\hline \multirow{4}{*}{100} & 00 & $a|0\rangle+b e^{i\left(\varphi_{1}+\varphi_{2}\right)}|1\rangle$ & $I$ \\
\hline & 01 & $a|0\rangle-b e^{i\left(\varphi_{1}+\varphi_{2}\right)}|1\rangle$ & $Z$ \\
\hline & 10 & $a|0\rangle-b e^{i\left(\varphi_{1}+\varphi_{2}\right)}|1\rangle$ & $Z$ \\
\hline & 11 & $a|0\rangle+b e^{i\left(\varphi_{1}+\varphi_{2}\right)}|1\rangle$ & $I$ \\
\hline \multirow{4}{*}{101} & 00 & $a|1\rangle+b e^{i\left(\varphi_{1}+\varphi_{2}\right)}|0\rangle$ & $X$ \\
\hline & 01 & $a|1\rangle-b e^{i\left(\varphi_{1}+\varphi_{2}\right)}|0\rangle$ & $Z X$ \\
\hline & 10 & $a|1\rangle-b e^{i\left(\varphi_{1}+\varphi_{2}\right)}|0\rangle$ & $Z X$ \\
\hline & 11 & $a|1\rangle+b e^{i\left(\varphi_{1}+\varphi_{2}\right)}|0\rangle$ & $X$ \\
\hline \multirow{4}{*}{110} & 00 & $a|0\rangle+b e^{i\left(\varphi_{1}+\varphi_{2}\right)}|1\rangle$ & $I$ \\
\hline & 01 & $a|0\rangle-b e^{i\left(\varphi_{1}+\varphi_{2}\right)}|1\rangle$ & $Z$ \\
\hline & 10 & $a|0\rangle-b e^{i\left(\varphi_{1}+\varphi_{2}\right)}|1\rangle$ & $Z$ \\
\hline & 11 & $a|0\rangle+b e^{i\left(\varphi_{1}+\varphi_{2}\right)}|1\rangle$ & $I$ \\
\hline \multirow{4}{*}{111} & 00 & $a|1\rangle+b e^{i\left(\varphi_{1}+\varphi_{2}\right)}|0\rangle$ & $X$ \\
\hline & 01 & $a|1\rangle-b e^{i\left(\varphi_{1}+\varphi_{2}\right)}|0\rangle$ & $Z X$ \\
\hline & 10 & $a|1\rangle-b e^{i\left(\varphi_{1}+\varphi_{2}\right)}|0\rangle$ & $Z X$ \\
\hline & 11 & $a|1\rangle+b e^{i\left(\varphi_{1}+\varphi_{2}\right)}|0\rangle$ & $X$ \\
\hline
\end{tabular}

JRSP protocol for an arbitrary single-qubit state with unit success probability (i.e, $P_{s u c}^{N \geq 2}=1$ ).

In addition, our protocol is also flexible and convenient: depending on the need, we can assign a receiver after sharing the quantum channel and the receiver may not be sufficiently well equipped. What is more, based on 
our protocol, we can structure a practical network: Alice can be regard as a Service Provider, she just distribute EPR pairs and measure her qubits; Other participants (Bob 1, Bob 2,.., Bob N-1 and Charlie) can be regard as customers, while one of them is a receiver and the others are preparers. That is to say, with the help of Alice, arbitrary customer can be a receiver or preparer.

Acknowledgements This work is supported by the National Nature Science Foundation of China (Grant Nos. 61103235, 61170321, 61373016 and 61373131), the Priority Academic Program Development of Jiangsu Higher Education Institutions (PAPD), the State Key Laboratory of Software Engineering, Wuhan University(SKLSE2012-09-41), and the Practice Inovation Trainng Program Projects for the Jiangsu College Students (201310300018Z).

\section{References}

1. Bennett, C.H., Brassard, G.: Quantum cryptography: Public key distribution and coin tossing. In: Proceedings of IEEE International Conference on Computers, Systems and Signal Processing, 1984, 175-179. IEEE Press, New York(1984)

2. Bennett, C.H.: Quantum cryptography using any two nonorthogonal states. Phys. Rev. Lett. 68(21), 3121-3124 (1992).

3. Yang, J., Xu, B.J., Guo, H.: Source monitoring for continuous-variable quantum key distribution. Phys. Rev. A 86(4), 042314 (2012). doi:10.1103/PhysRevA.86.042314

4. Cleve, R., Gottesman, D., Lo, H.K.: How to share a quantum secret. Phys. Rev. Lett. 83(3), 648-651 (1999). doi:10.1103/PhysRevLett.83.648

5. Hillery, M., Buzek, V., Berthiaume, A.: Quantum secret sharing. Phys. Rev. A 59(3), 1829-1834 (1999). doi:10.1103/PhysRevA.59.1829

6. Wang, H. B.; Huang, Y. G.; Fang, X.; Gu, B.; Fu, D. S.: High-capacity three-party quantum secret sharing with single photons in both the polarization and the spatial-mode degrees of freedom. Int. J. Theor. Phys. 52(4), 1043-1051 (2013). doi:10.1007/s10773-0121418-x

7. Deng, F.G., Long, G.L., Liu, X.S.: Two-step quantum direct communication protocol using the Einstein-Podolsky-Rosen pair block. Phys. Rev. A 68(4), 042317 (2003). doi:10.1103/PhysRevA.68.042317

8. Liu, W.J., Chen, H.W., Li, Z.Q., Liu, Z.H.: Efficient quantum secure direct communication with authentication. Chin. Phys. Lett. 25(7), 2354-2357 (2008).

9. Liu, W.J., Chen, H.W., Ma, T.H., Li, Z.Q., Liu, Z.H., Hu, W.B.: An efficient deterministic secure quantum communication scheme based on cluster states and identity authentication. Chinese. Phys. B 18(10), 4105-4109 (2009). doi:10.1088/1674-1056/18/10/007

10. Bennett, C.H., Brassard, G., Crpeau, C., Jozsa, R., Peres, A., Wootters, W.K.: Teleporting an unknown quantum state via dual classical and Einstein-Podolsky-Rosen channels. Phys. Rev. Lett. 70(13), 1895-1899 (1993).

11. Bouwmeester, D., Pan, J.W., Mattle, K., Eibl, M., Weinfurter, H., Zeilinger, A.: Experimental quantum teleportation. Nature 390(6660), 575-579 (1997). doi:10.1038/37539

12. Long, L.R., Li, H.W., Zhou, P., Fan, C., Yin, C.L.: Multiparty-controlled teleportation of an arbitrary GHZ-class state by using a d-dimensional (N+2)-particle nonmaximally entangled state as the quantum channel. SCI. China. Phys. Mech. 54(3), 484-490 (2011). doi:10.1007/s11433-011-4246

13. Liu, W.J., Liu, C., Liu, Z.H., Liu, J.F., Geng, H.T.: Same Initial States Attack in Yang et al.'s Quantum Private Comparison Protocol and the Improvement. Int. J. Theor. Phys. 53(1), 271-276 (2013)

14. Liu, W.J., Liu, C., Wang, H.B., Jia, T.T.: Quantum Private Comparison: A Review. IETE Tech. Rev. 30(5), 439-445 (2013). doi:10.1007/s10773-013-1807-9

15. Huang, W., Wen, Q.Y., Liu, B., Gao, F., Sun, Y.: Robust and efficient quantum private comparison of equality with collective detection over collective-noise channels. SCI. China. Phys. Mech. 56(9), 1670-1678 (2013). doi:10.1007/s11433-013-5224-0 
16. Pati, A.K.: Minimum classical bit for remote preparation and measurement of a qubit. Phys. Rev. A 63(1), 014302 (2000).

17. Bennett, C.H., DiVincenzo, D.P., Shor, P.W., Smolin, J.A., Terhal, B.M., Wootters, W.K.: Remote State Preparation. Phys. Rev. Lett. 87(7), 077902 (2001).

18. Lo, H.-K.: Classical-communication cost in distributed quantum-information processing: A generalization of quantum-communication complexity. Phys. Rev. A 62(1), 012313 (2000).

19. Xia, Y., Song, J., Song, H.S.: Multiparty remote state preparation. J. Phys. B: At. Mol. Opt. Phys. 40(18), 3719-3724 (2007). doi:10.1088/0953-4075/40/18/011

20. An, N.B., Kim, J.: COLLECTIVE REMOTE STATE PREPARATION. Int. J. Quantum Inf. 6(5), 1051-1066 (2008). doi:10.1142/s0219749908004304

21. Chen, Q.Q., Xia, Y., Song, J., Nguyen, B.A.: Joint remote state preparation of a W-type state via W-type states. Phys. Lett. A 374(44), 4483-4487 (2010). doi:10.1016/j.physleta.2010.09.013

22. Luo, M.X., Chen, X.B., Ma, S.Y., Niu, X.X., Yang, Y.X.: Joint remote preparation of an arbitrary three-qubit state. Opt. Commun. 283(23), 4796-4801 (2010). doi:10.1016/j.optcom.2010.07.043

23. Nguyen, B.A.: Joint remote preparation of a general two-qubit state. J. Phys. B: At. Mol. Opt. Phys. 42(12), 125501 (2009). doi:10.1088/0953-4075/42/12/125501

24. Nguyen, B.A.: Joint remote state preparation via W and W-type states. Opt. Commun. 283(20), 4113-4117 (2010). doi:10.1016/j.optcom.2010.06.016

25. Xia, Y., Song, J., Song, H.S., Guo, J.L.: MULTIPARTY REMOTE STATE PREPARATION WITH LINEAR OPTICAL ELEMENTS. Int. J. Quantum Inf. 6(5), 1127-1134 (2008). doi:10.1142/s0219749908004328

26. Xiao, X.-Q., Liu, J.-M., Zeng, G.: Joint remote state preparation of arbitrary two-and three-qubit states. J. Phys. B: At. Mol. Opt. Phys. 44(7), 075501 (2011).

27. An, N.B., Bich, C.T., Van Don, N.: Deterministic joint remote state preparation. Phys. Lett. A 375(41), 3570-3573 (2011). doi:10.1016/j.physleta.2011.08.045

28. Xia, Y., Chen, Q.-Q., An, N.B.: Deterministic joint remote preparation of an arbitrary three-qubit state via EinsteinCPodolskyCRosen pairs with a passive receiver. J. Phys. A: Math. Theor. 45(33), 335306 (2012).

29. Yuan, W., Xin, J.: Deterministic joint remote state preparation of arbitrary two-and three-qubit states. Chinese. Phys. B 22(2), 020306 (2013).

30. Ming-Ming, W., Xiu-Bo, C., Yi-Xian, Y.: Deterministic Joint Remote Preparation of an Arbitrary Two-Qubit State Using the Cluster State. Commun. Theor. Phys. 59(5), 568 (2013).

31. Bich, C.T., Don, N.V., An, N.B.: Deterministic Joint Remote Preparation of an Arbitrary Qubit via Einstein-Podolsky-Rosen Pairs. Int. J. Theor. Phys. 51(7), 2272-2281 (2012). doi:10.1007/s10773-012-1107-9

32. Nielsen, M.A., Chuang, I.L.: Quantum Computation and Quantum Information. Cambridge University Press, (2010)

33. Segal, I.E.: Postulates for general quantum mechanics. The Annals of Mathematics 48(4), 930-948 (1947). 